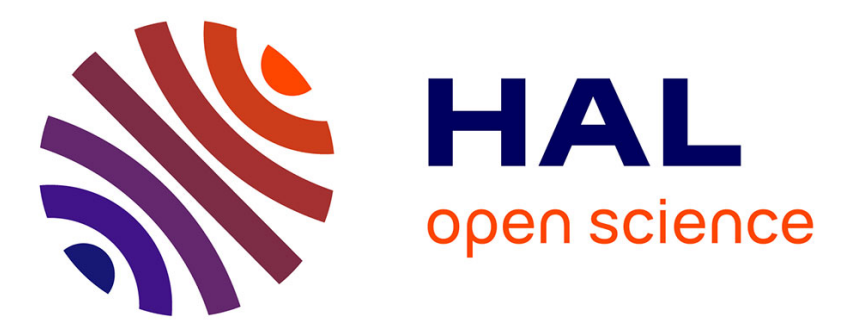

\title{
La résistance du piment (Capsicum annuum L.) à Phytophthora capsici Leon. XII. Influence de l'élicitation sur la résistance induite et l'accumulation du capsidiol
}

\author{
Paul-Michel Molot, Pierre Mas, Michèle Conus, Halina Ferrière
}

\section{To cite this version:}

Paul-Michel Molot, Pierre Mas, Michèle Conus, Halina Ferrière. La résistance du piment (Capsicum annuum L.) à Phytophthora capsici Leon. XII. Influence de l'élicitation sur la résistance induite et l'accumulation du capsidiol. Agronomie, 1984, 4 (9), pp.829-833. 10.1051/agro:19840904 . hal02726523

\section{HAL Id: hal-02726523 \\ https://hal.inrae.fr/hal-02726523}

Submitted on 2 Jun 2020

HAL is a multi-disciplinary open access archive for the deposit and dissemination of scientific research documents, whether they are published or not. The documents may come from teaching and research institutions in France or abroad, or from public or private research centers.
L'archive ouverte pluridisciplinaire HAL, est destinée au dépôt et à la diffusion de documents scientifiques de niveau recherche, publiés ou non, émanant des établissements d'enseignement et de recherche français ou étrangers, des laboratoires publics ou privés. 


\title{
La résistance du piment (Capsicum annuum L.) à Phytophthora capsici Leon. XII. - Influence de l'élicitation sur la résistance induite et l'accu- mulation du capsidiol.
}

Paul-Michel MOLOT, Pierre MAS, Michèle CONUS \& Halina FERRIÈRE

I.N.R.A., Station de Pathologie végétale, Centre de Recherches d'Avignon, B.P. 94, F 84140 Montfavet

RÉSUMÉ

\begin{abstract}
Un éliciteur fongique partiellement purifié (G5), issu de Phytophthora capsici et soluble dans l'eau, est mis au contact de tiges de piment adultes maintenues en survie. Trois techniques d'élicitation sont décrites. L'effet de protection le meilleur s'observe au niveau des tissus ayant été directement au contact de l'éliciteur : il se traduit par une diminution de longueur de tige nécrosée par $P$. capsici, diminution qui est plus importante chez la variété résistante " Phyo 636 " que chez la variété sensible « Yolo Wonder ».

L'éliciteur G5 n'induit pas de capsidiol dans les tiges traitées et, après contamination par $P$. capsici, il n'y a pas plus de capsidiol dans les tissus élicités que non élicités. D'autres mécanismes de défense sont envisagés.
\end{abstract}

Mots clés additionnels : Eliciteur, tige nécrosée, mécanismes de défense.

Resistance of pepper (Capsicum annuum L.) to Phytophthora capsici. XII. - Influence of elicitation on induced resistance and synthesis of capsidiol.

Pepper stems can be protected from Phytophthora capsici infection if they are treated with a partially purified and water soluble elicitor $\mathrm{G} 5$ which is obtained from the same fungus. Three methods of elicitation are described. The best protection effect was observed in tissues in direct contact with elicitor. Moreover the effect was greater with a resistant cultivar such as 'Phyo 636' than with a susceptible one 'Yolo Wonder'. The elicitor G5 did not induce capsidiol production in treated stems and, after inoculation with $P$. capsici, there was no more capsidiol in the elicited tissues than in the non elicited tissues. This may mean other defence mechanisms have to be considered.

Additional key words : Elicitor, stem necrosis, defence mechanism.

\section{INTRODUCTION}

En réponse à une agression parasitaire, de nombreuses plantes réagissent par la synthèse de phytoalexines (BAILEY \& DEVERALL, 1971 ; GARCIAARENAL et al., 1978 ; YOSHIKAWA et al., 1978 ; BAILEY et al., 1980).

Chez le piment, l'attaque des tiges par Phytophthora capsici Leon. déclenche, au front de progression des hyphes, l'accumulation de plusieurs phytoalexines, notamment de capsidiol (MOLOT et al., 1976, 1981). Cependant, en dépit d'une activité biologique indéniable (Molot et al., 1980a), cette substance ne semble être à la base ni de la spécificité de la résistance (Molot et al., 1977), ni de la résistance induite mise en évidence par une $2^{\mathrm{e}}$ contamination (MOLOT et al., 1982 ; MOLOT \& MAS, 1983).

En absence de contamination, il est maintenant bien établi que certaines substances, provenant des surfaces cellulaires des champignons, sont capables d'induire chez les plantes la formation de phytoalexines (AYERS et al., 1976 ; ALBERSHEIM \& VALENT, 1978 ; KEEN \& LEGRAND, 1980 ; RICCI, 1980). Ces substances, appelées éliciteurs, stimulent la synthèse de novo des enzymes impliquées dans le métabolisme des phytoalexines (DIXON \& LAMB, 1979 ; HAHLBROCK et al., 1981). On sait aussi que les éliciteurs issus de divers champignons peuvent déclencher, chez une même plante, la synthèse d'une phytoalexine (HAHN \& ALBERSHEIM, 1978) et qu'un éliciteur donné peut induire, chez 
diverses plantes, la formation de différentes phytoalexines (CLINE et al., 1978).

Dans le cas du piment, on a montré que les filtrats de culture de $P$. capsici induisent au niveau des tiges (SIMON, 1977) et des organes foliaires (MOLOT et al., 1980 b) la synthèse de capsidiol. Ils renferment donc des éliciteurs que l'on tente actuellement de séparer selon le mode de fractionnement $G$ (Molot et al., 1980c).

Habituellement, la mise au contact de l'éliciteur avec le végétal se fait au niveau des organes foliaires. Ici, elle est réalisée sur tiges de piment qui, dans les conditions naturelles, sont avec les racines les cibles privilégiées de $P$. capsici. En procédant ainsi, nous vérifierons tout d'abord la possibilité d'éliciter des tissus différents. De plus, étant donné le caractère systémique des effets de protection observés, nous ferons absorber l'éliciteur soit au site même de la contamination, soit en un point éloigné de ce dernier. Les tiges contaminées ou non seront alors prélevées en vue de rechercher les quantités de capsidiol présentes.

\section{MATÉRIEL ET MÉTHODES}

\section{A. Matériel végétal et cryptogamique}

Nous utilisons les 2 variétés de piment : «Yolo Wonder " sensible et «Phyo 636 » résistante à $P$. capsici. Ce matériel est cultivé sous serre jusqu'au stade d'apparition des boutons floraux. Les contaminations artificielles sont réalisées avec la souche 15 de $P$. capsici cultivée sur milieu V8.

\section{B. Techniques d'élicitation et de contamination arti- ficielle}

Après prélèvement des tiges, celles-ci sont effeuillées soit partiellement (on ne laisse alors subsister que le «bouquet » de feuilles terminales), soit totalement. Dans ce dernier cas, la tige est réduite à une «baguette " d'environ $20 \mathrm{~cm}$ dont les 2 extrémités sont sectionnées.

Bien que n'étant pas la plus performante, la fraction G5 issue de $P$. capsici souche 15 (Molot et al., 1980c) a été choisie en raison de sa solubilité dans l'eau et des réserves relativement importantes dont nous disposions. En effet, contrairement aux microtests réalisés sur organes foliaires, les manipulations décrites ici exigent d'assez grands volumes de solution élicitrice.

Trois techniques d'absorption de cet éliciteur sont mises en comparaison :

- Technique A : trempage dans G5 du «talon» de la tige totalement effeuillée.

- Technique B : trempage dans G5 du «talon» de la tige possédant un « bouquet » de feuilles terminales.

- Technique $\mathrm{C}$ : trempage dans G5 de la section supérieure de la tige totalement effeuillée (« baguette » retournée). Dans ce cas les tissus externes sont directement au contact de l'éliciteur.

La concentration de l'éliciteur est de $0,5 \mathrm{mg} / \mathrm{ml}$ d'eau et la quantité de liquide est ajustée de façon à recouvrir $2 \mathrm{~cm}$ de tige.
Après $24 \mathrm{~h}$ de trempage, on procède ou non à la contamination artificielle des tiges par une pastille de culture mycélienne sur milieu gélosé de $P$. capsici $(\varnothing=4 \mathrm{~mm})$. Le dépôt de cet implant s'effectue en haut de la tige, sur une section rafraîchie :

+ après ablation du " bouquet » de feuilles terminales (technique $B$ ),

+ après retrait d'un tronçon de $1 \mathrm{~mm}$ d'épaisseur (technique $\mathrm{A}$ et $\mathrm{C}$ ).

Les «baguettes » $C$ retrouvent alors leur polarité normale. L'inoculum est ensuite coiffé par un petit capuchon de papier aluminium servant de chambre humide et les «talons » de toutes les tiges sont remis dans l'eau.

Les plantes témoins où l'éliciteur est remplacé par l'eau subissent les mêmes traitements.

Les notations consistent à mesurer, tous les $2 \mathrm{j}$, les longueurs de tiges nécrosées.

\section{Dosages de capsidiol}

Les prélèvements de tiges sont effectués $5 \mathrm{j}$ après le début de l'élicitation (on sait en effet que le maximum d'accumulation du capsidiol se situe environ 4 à $5 \mathrm{j}$ après le stimulus de départ) et concernent des tiges contaminées ou non.

Sur chaque tige contaminée, juste sous le front de progression des hyphes, on découpe un tronçon de $1 \mathrm{~cm}$ de hauteur. Chez les tiges non contaminées, on débite, après rafraîchissement et juste sous la section supérieure, un cylindre de $1 \mathrm{~cm}$.

Après broyage des tissus dans l'éthanol à 50 p. 100 , les extraits sont chromatographiés en couche mince et le capsidiol est révélé biologiquement, le diamètre des zones d'inhibition de Cladosporium herbarum étant proportionnel aux quantités de capsidiol (MOLOT et al., 1976 ; MOLOT et al., 1980b et c). Signalons que 10 échantillons identiques (correspondant à du matériel élicité et infecté, donc riche en capsidiol) analysés après traitement par l'éthanol donnent des résultats très comparables avec un coefficient de variation voi$\sin$ de 7 p. 100 .

\section{RÉSULTATS}

En l'absence de contamination, il n'y a pas de synthèse de capsidiol, quelle que soit la technique d'induction utilisée, c'est-à-dire que les tissus aient été ou non en contact direct avec l'éliciteur.

Si l'on pratique une contamination artificielle, les longueurs de tiges nécrosées et les quantités de capsidiol trouvées au front de progression des hyphes mettent en évidence plusieurs points intéressants (tabl. 1) :

- Longueurs de tiges nécrosées:

Le trempage dans l'éliciteur du « talon » de la tige totalement effeuillée (technique A) ne modifie pas la longueur de nécrose provoquée par $P$. capsici. Par contre, si on laisse un «bouquet 》 de feuilles (technique B) jouant sans doute le rôle de «tire-sève ", on obtient, uniquement chez la variété résistante «Phyo 636 », une différence significative dans les longueurs de tissu nécrosé par le champignon, les tiges « élicitées " paraissant moins sensibles. 
TABLEAU 1

Action de G5 15 sur la sensibilité des tiges de piment à Phytophthora capsici (souche 15) et sur l'accumulation du capsidiol. (Lectures et dosages, 5 jours après le début de l'élicitation.)

Action of G5 15 on susceptibility of pepper stems to Phytophthora capsici (strain 15) and on synthesis of capsidiol (5 days after elicitation outset). after elicitation outset).

\begin{tabular}{|c|c|c|c|c|c|c|c|}
\hline \multirow{3}{*}{$\begin{array}{l}\text { Technique } \\
\text { utilisée }\end{array}$} & & \multicolumn{3}{|c|}{ Yolo Wonder } & \multicolumn{3}{|c|}{ Phyo 636} \\
\hline & & \multirow{2}{*}{$\frac{\text { Témoin }}{\varnothing}$} & \multicolumn{2}{|c|}{ Contaminé } & \multirow{2}{*}{$\frac{\text { Témoin }}{\varnothing}$} & \multicolumn{2}{|c|}{ Contaminé } \\
\hline & & & $\mathrm{L}$ & $\varnothing$ & & $\mathrm{L}$ & $\varnothing$ \\
\hline \multirow{2}{*}{ A } & Eau & 0 & $41,3 \pm 4,1$ & $24 \pm 2,2$ & 0 & $23,1 \pm 2,6$ & $30 \pm 3,1$ \\
\hline & G5 15 & 0 & 43,0 & 24 & $\epsilon$ & 22,8 & 29 \\
\hline \multirow{2}{*}{ B } & Eau & $\epsilon$ & 40,1 & 25 & 0 & 31,5 & 28 \\
\hline & G5 15 & 0 & 39,7 & 23 & 0 & 25,6 & 29 \\
\hline \multirow{2}{*}{$\mathrm{C}$} & Eau & 0 & 42,2 & 20 & $\epsilon$ & 18,7 & 28 \\
\hline & G5 15 & $\epsilon$ & 30,2 & 22 & 0 & 10,1 & 27 \\
\hline
\end{tabular}

$A=$ Tige totalement effeuillée dont le «talon " trempe dans l'éliciteur.

Entirely defoliated stems dipped into elicitor at base and inoculated at top.

$B=$ Tige conservant un "bouquet " terminal de feuilles pendant la durée du trempage du " talon " dans l'éliciteur.

Partially defoliated stems (with few leaves at the top) dipped into elicitor at base and inoculated at top.

$C=$ Tige totalement effeuillée et dont la section supérieure trempe dans l'éliciteur avant d'être retournée et contaminée.

Entirely defoliated stems dipped into elicitor at top and inoculated at top.

$L=$ Longueur moyenne en $m m$ de tige nécrosée.

Average length $(\mathrm{mm})$ of stem necrosis.

$\varnothing=$ Diamètre en $\mathrm{mm}$ de la zone d'inhibition de Cladosporium herbarum (estimation de la quantité de capsidiol).

Diameter ( $\mathrm{mm}$ ) of $\mathrm{C}$. herbarum inhibition with chromatographic bioassay (estimated quantity of capsidiol).

Avec la technique $C$, les tiges des 2 variétés traitées par G5 sont plus résistantes que les tiges témoins ; chez "Phyo 636 », la sensibilité est de 54 p. 100 par rapport au témoin, chez "Yolo Wonder» de 71 p. 100.

- Quantités de capsidiol présentes au front de progression des hyphes :

Après contamination, le capsidiol peut être mis en évidence dans les tissus, mais il n'y a aucune différence significative entre tiges "élicitées» et tiges «non élicitées». On remarquera seulement que «Phyo 636 » synthétise plus de capsidiol que « Yolo Wonder ».

\section{DISCUSSION, CONCLUSION}

Après la mise en contact avec un éliciteur purifié, les tiges de piment non contaminées ne synthétisent pas de capsidiol. Ce résultat avait déjà été obtenu avec des organes foliaires de piment en survie sur du filtrat de culture brut : si le filtrat est dilué de moitié ou s'il n'est laissé en contact avec les tissus que peu de temps (environ $2 \mathrm{~h}$ ), il n'y a pas synthèse de capsidiol (MOLOT et al., 1980b). Un phénomène analogue se retrouve quand on dépose sur cotylédons 2 fractions élicitrices de même origine, l'une ayant été soumise à dialyse, l'autre non : après action de la $1^{\text {re }}$ fraction, on ne détecte plus de capsidiol (MOLOT et al., 1980c).

La contamination artificielle des extrémités supérieures de tige ayant trempé directement dans l'éliciteur révèle une faible réceptivité des tissus à $P$. capsici.
L'effet est plus net chez la variété résistante que chez la variété sensible. Sur feuilles adultes, maintenues en survie sur l'eau et élicitées par une goutte de la fraction G4 (rétentat insoluble homologue de G5), nous avons déjà signalé un phénomène de protection analogue (MOLOT \& MAS, 1983).

L'élément le plus remarquable semble résider dans le fait qu'après contamination, il n'y ait pas plus de capsidiol dans les tiges élicitées que dans les tiges témoins. Ce résultat est en contradiction avec les travaux de plusieurs auteurs. Chez le haricot, les éliciteurs induisent toujours l'accumulation de phaséoline, aussi bien chez les plantes saines que chez les plantes infectées par Uromyces phaseoli (HOPPE et al., 1980). Dans le cas du riz, l'acide 2,2-dichloro-3,3-diméthyl cyclo propane carboxylique ne stimule pas lui-même la production de phytoalexines (momilactones A et B) mais, après infection de la plante par Piricularia oryzae, il augmente considérablement la capacité du riz à synthétiser ces substances (CARTWRIGHT et al., 1977). 1977).

Pour expliquer l'absence de capsidiol, on peut formuler quelques hypothèses :

- différents travaux, en particulier ceux réalisés sur les Phytophthora de la pomme de terre (BOSTOCK et al., 1982) et du soja (KEEN et al., 1983) ont montré qu'après purification les éliciteurs perdent souvent une partie, sinon la totalité de leur activité inductrice de phytoalexines ;

- la décapitation de la tige entraîne des modifications physiologiques au niveau de la balance hormonale. Dans ces conditions, il peut y avoir, dans la 
chaîne métabolique, des déviations qui bloquent la formation de phytoalexines ou aboutissent à des changements structuraux. De tels phénomènes ont été signalés chez l'aubergine (WARD et al., 1975) et chez la pomme de terre (STOESSL \& STOTHERS, 1981).

On peut imaginer également qu'après action de l'éliciteur d'autres phytoalexines que le capsidiol apparaissent. On a trouvé récemment dans les fruits issus de Capsicum annuum des quantités importantes de capsicannol (ADIKARAM et al., 1982). Mais, à notre connaissance, cette substance n'a jamais été mise en évidence dans les tiges de piment.

Chez le piment, l'induction de résistance par des éliciteurs fongiques (G4 ou G5) n'étant pas accompagnée de synthèse de capsidiol, il convient de rechercher d'autres mécanismes de défense. Plusieurs hypothèses ont été envisagées et, sur d'autres modèles, certaines d'entre elles ont été clairement démontrées. Avec le couple melon-Colletotrichum lagenarium, les éliciteurs sont capables d'induire dans l'hôte l'accumulation de glycoprotéines pariétales riches en hydroxyproline (ROBY, 1981). Ce même auteur a également mis en évidence, après élicitation, une augmentation du dégagement d'éthylène et des quantités d'inhibiteurs protéasiques. Par ailleurs, les éliciteurs sont en mesure d'exercer des effets physiologiques importants notamment en affectant la perméabilité cellulaire : avec des suspensions cellulaires de soja, l'addition d'éliciteur inhibe l'absorption de nitrate (EBEL et al., 1976) ; chez le melon, il y a réduction importante de l'assimilation des acides aminés marqués (ROBY, 1981). De façon plus précise, on sait aussi que les éliciteurs peuvent stimuler l'activité des ARN messagers des inhibiteurs protéasiques (NELSON et al., 1981). Enfin, sur plantules de piment, les processus d'élicitation entraînent une augmentation de la concentration en protéines totales parmi lesquelles certaines fractions d'activité peroxydasique répondent avec une amplitude décuplée (COUlOMB et al., 1983).

Les éliciteurs sont capables d'induire chez les plantes une réaction de défense à l'égard d'un parasite. Cette réaction fort complexe est sans doute le résultat d'une réorientation du métabolisme protéique des cellules-hôtes. Dans la mesure où il n'y a pas synthèse de capsidiol, le couple piment-Phytophthora peut constituer un modèle d'approche intéressant pour des travaux ultérieurs.

\section{RÉFÉRENCES BIBLIOGRAPHIQUES}

Adikaram N. K. B., Brown A. E., Swinburne T. R., 1982. Phytoalexin involvement in the latent infection of Capsicum annuum $\mathrm{L}$. fruit by Glomerella cingulata (Stonem). Physiol. Plant Pathol., 21, 161-170.

Albersheim P., Valent B., 1978. Host-pathogen interactions in plants. Plants, when exposed to oligosaccharides of fungal origin, defend themselves by accumulating antibiotics. J. Cell. Biol., 78, 627-643.

Ayers A. R., Ebel J., Valent B., Albersheim P., 1976. Hostpathogen interactions. X. Fractionation and biological activity of an elicitor isolated from the mycelial walls of Phytophthora megasperma var. sojae. Plant Physiol., 57, 760-765.

Bailey J. A., Deverall B. J., 1971. Formation and activity of phaseollin in the interaction between bean hypocotyls (Phaseolus vulgaris) and physiological races of Colletotrichum lindermuthianum. Physiol. Plant Pathol., 1, 435-449.

Bailey J. A., Rowell P. M., Arnold G. M., 1980. The temporal relationship between host cell death, phytoalexin accumulation and fungal inhibition during hypersensitive reactions of Phaseolus vulgaris to Colletotrichum lindemuthianum. Physiol. Plant Pathol., 17, 329-339.

Bostock R. M., Laine R. A., Kuc J. A., 1982. Factors affecting the elicitation of sesquiterpenoid phytoalexin accumulation by eicosapentaenoic and arachidonic acids in potato. Plant Physiol., 70, $1417-1424$.

Cartwright D., Langeake P., Pryce R. J., Leworthy D. P., Ride J. P., 1977. Chemical activation of host defence mechanisms as a basis for crop protection. Nature (London), 267, 511-513.

Cline K., Wade M., Albersheim P., 1978. Host-pathogen interactions. XV. Fungal glucans which elicit phytoalexin accumulation in soybean also elicit the accumulation of phytoalexins in other plants. Plant Physiol., 62, 918-921.

Coulomb C., Molot P. M., Mas P., Coulomb J. Ph., Bounias M., 1983. Analyse des variations de l'activité peroxydasique soluble (E.C. 1-11-1-7) dans les feuilles de Capsicum annuum (piment) au cours d'une induction systémique de résistance à Phytophthora capsici (mildiou). C.R. Acad. Sc. Paris, t. 296, 225-230.
Dixon R. A., Lamb C. J., 1979. Stimulation of de novo synthesis of L-phenyl alanine ammonia-lyase in relation to phytoalexin accumulation in Colletotrichum lindemuthianum elicitor-treated cell suspension cultures of French bean (Phaseolus vulgaris). Biochim. Biophys. Acta, 586, 453-463.

Ebel J., Ayers A. R., Abersheim P., 1976. Host-pathogen interactions. XII. Response of suspension cultured soybean cells to the elicitor isolated from Phytophthora megasperma var. sojae, a fungal pathogen of soybeans. Plant Physiol., 57, 775-779.

Garcia-Arenal F., Fraille A., Sagasta E. M., 1978. The multiple phytoalexin response of bean (Phaseolus vulgaris) to infection by Botrytis cinerea. Physiol. Plant Pathol., 13, 151-156.

Hahlbrock K., Lamb C. J., Purwin C., Ebel J., Fautz E., Schafer E., 1981. Rapid response of suspension-cultured parsley cells to the elicitor from Phytophthora megasperma var. sojae. Plant Physiol, 67, 768-773.

Hahn M. G., Albersheim P., 1978. Host-pathogen interactions. XIV. Isolation and partial characterization of an elicitor from yeas extract. Plant Physiol., 62, 107-111.

Hoppe H. H., Humme B., Heitefuss R., 1980. Elicitor induced accumulation of phytoalexins in healthy and rust infected leaves of Phaseolus vulgaris. Phytopathol. Z., 97, 85-88.

Keen N. T., Legrand M., 1980. Surface glycoproteins : evidence that they may function as the race specific phytoalexin elicitors of Phytophthora megasperma f. sp. glycinea. Physiol. Plant Pathol., 17, 175-192.

Keen N. T., Yoshikawa M., Wang M. C., 1983. Phytoalexin elicitor activity of carbohydrates from Phytophthora megasperma $f$. $s p$. glycinea and other sources. Plant Physiol., 71, 466-471.

Molot P. M., Clerjeau M., Nourrisseau J. G., Ricci P., 1976. La résistance du piment (Capsicum annuum) à Phytophthora capsici. III. Etude, sur extraits de tiges sensibles et résistantes, du pouvoir antifongique induit par la contamination. Ann. Phytopathol., 8 (4), 399-407. 
Molot P. M., Clerjeau M., Mas P., Ricci P., 1977. Rôle du capsidiol dans la résistance génétique du piment (Capsicum annuum) au Phytophthora capsici. C.R. $3^{e}$ Congrès Eucarpia Piment, Avignon, 5-8 juillet 1977, 137-146.

Molot P. M., Mas P., Hilario A. L., 1980a. La résistance du piment (Capsicum annuum) à Phytophthora capsici. VI. Pouvoir inhibiteur du capsidiol sur la croissance et l'activité pectinolytique d'isolats d'agressivité variable. Ann. Phytopathol., 12 (1), 1-9.

Molot P. M., Mas P., Conus M., Ferrière H., 1980b. La résistance du piment à Phytophthora capsici. VII. Protection des organes foliaires après mise en survie sur un filtrat de culture du parasite. Caractérisation des conditions d'expression du phénomène. Ann. Phytopathol., 12 (2), 95-107.

Molot P. M., Staron T., Mas P., 1980c. La résistance du piment à Phytophthora capsici. VIII. Induction de résistance et de capsidiol chez Capsicum annuum avec des fractions obtenues à partir de filtrats de culture et du mycélium de Phytophthora capsici. Ann. Phytopathol., 12 (4), 379-387.

Molot P. M., Mas P., Conus M., Ferrière H., Ricci P., 1981. Relations between capsidiol concentration, speed of fungal invasion and level of induced resistance in cultivars of pepper (Capsicum annuum) susceptible or resistant to Phytophthora capsici. Physiol. Plant Pathol., 18, 379-387.

Molot P. M., Mas P., Ricci P., 1982. La résistance du piment (Capsicum annuum) d̀ Phytophthora capsici. IX. Distribution spatiotemporelle du capsidiol dans les tiges infectées. Agronomie, 2 (9), 865-869.
Molot P. M., Mas P., 1983. La résistance du piment (Capsicum annuum) à Phytophthora capsici. X. Influence de la température sur l'accumulation du capsidiol et les variations de l'induction de résistance. Agronomie, 3 (1), 39-44.

Nelson C. E., Walker-Simmons M., Ryan C. A., 1981. Regulation of proteinase inhibitor synthesis in tomato leaves. In vitro synthesis on inhibitors I et II with mRNA from excised leaves induced with P.I.I.F. Plant Physiol., 67, 841-844.

Ricci P., 1980. Induced resistance in carnation infected by Phytophthora parasitica. Active Defence Mechanisms in Plants. Nato Advanced Study Institute, vol. 37.

Roby D., 1981. Induction par des éliciteurs fongiques, via l'éthylène, de réactions associées à la défense des plantes. Thèse Doct. $3^{\mathrm{e}}$ cycle, Univ. Toulouse, $67 \mathrm{p}$.

Simon M. F., 1977. Mise au point d'une technique d'induction de phytoalexines chez le piment. Mémoire de fin d'études. U.B.O. Brest.

Stoessl A., Stothers J. B., 1981. A carbon-13 biosynthetic study of stress metabolites from potatoes : the origin of isolubimin. Can. J. Bot., 59 (5), 637-639.

Ward E. W. B., Unwin C. H., Hill J., Stoessl A., 1975. Sesquiterpenoid phytoalexins from fruits of eggplants. Phytopathology, 65 (2), 859-863.

Yoshikawa M., Yamauchi K., Masago H., 1978. Glyceollin : its role in restricting fungal growth in resistant soybean hypocotyls infected with Phytophthora megasperma var. sojae. Physiol. Plant Pathol., 12, 73-82. 